\title{
Non-Operating Room Anesthesia: An Overview
}

\author{
Yeliz Kılıç \\ Department of Anesthesiology and Reanimation, Eskișehir Osmangazi University School of Medicine, Eskișehir, Turkey
}

ORCID iD of the author: Y.K. 0000-0003-1446-7747.

Cite this article as: Kılıç Y. Non-operating Room Anesthesia: An Overview. Cyprus J Med Sci 2020; 5(2): $171-5$.

\begin{abstract}
Today, an increasing number of diagnostic and therapeutic procedures are performed in specialized units outside the classical operating room, such as endoscopy units, interventional radiology, neurology and cardiology laboratories. The main reasons of this global trend are recent advances in medical technology, increased knowledge of disease pathogenesis, and some financial factors. Additionally, number and complexity of these procedures are rapidly raising throughout the world. In parallel, the importance of anesthesia management of such patients, also known as non-operating room anesthesia, has become better understood in recent years. In this review, we aimed to discuss the potential difficulties of non-operating room anesthesia, preprosedural patient preparation, intraoperative anesthesia applications, and postprosedural patient care in the light of the current literature.
\end{abstract}

Keywords: Complication, interventional procedure, non-operating room anesthesia, management

\section{INTRODUCTION}

Thanks to advances in medical technology, increased knowledge of disease pathogenesis, and some financial factors, a significant number of diagnostic and therapeutic procedures are strictly being performed outside the traditional operating room (OR) such as endoscopy suites, cardiac catheterization laboratories, and invasive radiology units $(\mathrm{I}, 2)$. Those procedures were previously performed in selected patients and indications whereas potentially risky groups with medically unstable conditions, including geriatric, pediatric, and emergent patients, are now increasingly treated in these nonOR locations. In parallel to this global trend, anesthesia management of the patients undergoing such procedures, namely non-operating room anesthesia (NORA), has become an important key factor for patient safety and the success of the procedure. Several national anesthesia and reanimation societes, such as The American Society of Anesthesiologists (ASA), have published their clinical guidelines in order to make a standardization in routine practice $(3,4)$. However, more novel procedures are being included daily practice with each passing day. This situation, naturally, poses new challenges for anesthesiologists. Therefore, NORA should be considered as a special subunit of anesthesia practice.

In this review, we aimed to report both avantages and disadvantages of NORA, to discuss periprocedural patient assessment, and to highlight the potential key points of the anesthetic management, relevant with current literature.

\section{Potential Challenges of NORA}

In general, many anesthesiologists do not feel themselves safely when they have to work in any places outside the classical OR. Therefore, working in such places are usually not preffered by anesthesiologists. The most important reasons of this situation are unfamiliarity with the enviroment, unavilability of some critical equipments and supplies, and limited help from other anesthesiologists in case of emergent conditions (5-7). Increasing complexity of the procedures and patient expectancy are also among the potential concerns of anesthesiologists $(8,9)$. In addition, patients scheduled for such procedures are often selected by the severity of their disease, which prevents them from undergoing a major surgical procedure in the traditional OR. These patients are sometimes more medically compromised and less optimized in comparison to OR patients. Thus, anesthetic management of such patients is considered as more difficult or less safely.

Sufficiency of staff personnel in those locations may be another problematic issue. Those personnels may be less familiar with the overall management of anesthetized patients $(I 0, I I)$. They may even have limited medical background. However, it is a reality that assistance of trained personnel is an important factor at all stages of patient management, particulary for emergency situations (12). 


\section{Preprocedural Evaluation of Patients}

\section{Basic data and goals}

Preprocedural evaluation of patients is a critical step in the management of these patients. With the growing number of non-OR procedures, a significant number of patients now require assessment before their procedural date. The reality is that many anesthetic evaluations are today performed on the day of the procedure. However, this may be more costly by increasing the risk of procedural cancellations or delays (I3). Therefore, a standard protocol for preprocedural evaluation of non-OR patients should be established in all medical centers where non-OR procedures are routinely performed (14). It should be also stated here that this kind of preprocedural screening should be similar with patients scheduled for surgery at OR (12).

There are several basic goals for anesthetic management in non-OR procedures (Table I). The first goal of the preprocedural screening should include a comprehensive evaluation of patients, which should not be limited to only the past medical, surgical, anesthesia, family, medication and allergic reactions but also include potential procedure-related problems (I5). A complete physical examination is also an essential part of the preprocedural assessment. In the evaluation of pediatric population, the anesthesiologist should be attentive to the dynamics of the patient parent/guardian interaction (12). Similar to preanesthesia assessment of classic OR patients, anesthesiologist should be encourge to consult other medical services since these patients may have accompanying diseases.

Preparation and education of the patient, parent or guardian are also among the most important parts of the preprocedural evaluation (16). Patients and/or their relatives should be informed about the process in detail, and their written consent forms must be taken from all. In pediatric population, it has been showed that written information enhances a parent's understanding (I7).

\section{System evaluations and diagnostic testing}

A complete examination of the patient should be a mandatory strategy to conducting a safe procedure. System evaluations, in-

\section{Main Points:}

- Increased number and complexity of interventional procedures at specific places outside the classical operating room in recent years have clearly showed the importance of NORA.

- NORA carries several potential challenges including unfamiliarity of anesthesiologists with the enviroment, unavilability of some critical equipments and supplies, limited help from other anesthesiologists in case of emergent conditions, and sufficiency of staff personnel.

- Design of locations performed NORA procedures, careful preprocedural patient evaluation, appropriate anesthesia techniques, and closed postprocedural patient care play crurical role for the success of these procedures.

- A closed collaboration between anesthesiologists, proceduralists, other staff personnels, and hospital administrators is a mandatory step to minimizing the potential challenges and providing good results. cluding airway assessment, pulmonary, cardiac, and neurologic examinations must be performed in all non-OR patients. As a general approach, preprocedural diagnostic testing should be individualized based on patient risk factors and the procedure itself. Routine preoperative tests have rarely been shown to impact patient management (18). These tests should only be ordered if they will impact the care provided. Baseline laboratory tests including blood count, hepatic and renal function parameters, coagulation profile, and blood typing should be drawn for patients with potential bleeding risks or renal injury risk (14). However, it is a reality that classical laboratory tests, chest X-ray, and electrocardiography (ECG) are routinely performed in most medical centers, due to the legal concerns. Several basic rules have been determined by major international guidelines (Table 2).

Airway status should be clearly defined with using standard scoring systems such as mallampati score. Additionally, thorough airway examination with assessment of mouth opening, thyromental distance, dental condition, and neck mobility should be performed and recorded.

Pulmonary complications can contribute significantly to perioperative morbidity and mortality. Hypoxia and desaturation are the most common respiratory events during the periprocedural period. Smoking, lung disease, older age, malnutrition and long duration of anesthesia are the well known respiratory risk factors, especially for patients undergoing general anesthesia (1921). Chronic obstructive pulmonary disease, higher ASA scores, impaired sensorium, and obstructive sleep apnea are the other medical risk factors. Pulmonary function tests and/or consultation from department of pulmonary diseases may be useful in patients with bronchial asthma or chronic obstructive pulmonary disease. Current medications of the patients who have a pulmonary disease also carry great importance in the management

TABLE I. Basic goals for procedural anesthesia

Minimizing psychological discomfort and anxiety for both the patient and/or family

Selection of accurate anesthetic technique

Application of accurate and adequate medications

Careful monitoring during the anesthetic management

Optimizing pain management

Control movement to improve safety of invasive procedures

TABLE 2. Basic rules of radiological and hematological testing in preprocedural evaluation outside of the OR $(18,19)$

ECG is indicated in patients above 50 years, and is valid for six months provided no interval change.

Chest radiography is not mandatory at any age.

Complete blood count and electrolyte panel as indicated by history, and valid for six months.

Blood glucose check for all diabetic patients.

Potassium check for patients with end stage renal disease.

Pregnancy test on the day of the procedure is indicated for women of child-bearing age who had no documented hysterectomy.

An indication should be made for all tests ordered.

Tests already in the record are acceptable. 
of possible pulmonary complications. In this context, pulmonary inhalers should not be held on the day of procedure. Contrary, these inhalers should be administered before the procedure to avoid or minimizing the potential pulmonary events (19).

Cardiac evaluation is of great importance in these patients. Using a cardiac risk assessment algorithm is usually recommended during the preprocedural setting (22). Presence of ischemic heart disease, cardiac failure, diabetes, stroke, peripheral vascular disease, and renal insufficiency (creatinine above $2 \mathrm{mg} / \mathrm{dl}$ ), high-risk surgery, and older age have been determined as risk factors (23,

TABLE 3. Fasting status in NORA patients (24)

\begin{tabular}{|lcc|}
\hline Type of oral feeding & Patient group & duration \\
\hline Breastfeeding & Newborns and infants & 4 hours \\
Baby food or solid food & Newborns and infants & 6 hours \\
Clear fluids & Adult and pediatrics & 2 hours \\
Light solid meal & Adult & 6 hours \\
Solid or heavy meal & Adult & 8 hours \\
\hline
\end{tabular}

TABLE 4. Special clinical conditions indicating risky anesthesia management (28)

Emergent procedures

Complex, very long, or risky (ie, bleeding) procedures

Patients under 18 years old

Pregnancy

Uncooperative or patients with agitated or altered mental status

Patients with significant cardiac, respiratory, or systemic comorbidities ASA class III-IV

Increased risk for airway obstruction (morbid obesity, sleep apnea, mallampati score 3-4, Micrognathia, retrognathia, small mouth opening $(<3 \mathrm{~cm}$ in an adult)

Intolerance to the medications used for sedation (ie, benzodiazepines and narcotics)

Previous problems with sedation or with anesthesia

History of long-term use/abuse of benzodiazepines, narcotics, alcohol, or neuropsychiatric

medications

TABLE 5. Common potential problems that can occur during NORA* Respiratory events (ie, hypoxia, desaturation)

Hypothermia

Hypovolemia

Airway difficulties

Aspiration of gastric content,

Side effects of drugs or anaphylaxis

Postoperative nausea and vomiting

Procedure related complications (ie, bleeding, gastrointestinal perforation)

Harm to the anesthesiologist (ie, waste inhalational anesthetics and exposure to radiation)

Death

*Adopted from the study by Melloni C. (29)
24). Additional cardiac testing has been recommended for patients with unstable coronary syndrome (myocardial infarction and unstable angina), decompensated heart failure, significant dysrhythmias (high degree or Mobitz II AV block, $3^{\text {rd }}$ degree AV block), and severe valvular disease (19). The current medications, especially anticoagulants, antiplatelets, and antihypertensives, should be also examined in those patients because some drugs should be continued the day of procedure while others should be held on before the procedure. Stopping or continuing such medications should be discussed with the relevant departments during the preprocedural setting. The current trend is that most of these drugs, such as aspirin, should be continued unless there is a patient/procedure-related contraindication. There is a controversy on the preprocedural use of beta-blockers. However, the general approach on this issue is that beta-blocker drugs, especially metoprolol, should not be introduced immediately before the procedure but that beta-blockade should be continued in patients who are chronically receiving these medications. Angiotension converting enzyme inhibitors and angiotension receptor blockers should be held 12-24 hours before the procedure due to the concerns of causing vasoplegia. Diuretics should be also held on the day of the procedure whereas other antihypertensive drugs should be continued during the periprocedural period (19). Finally, endocarditis prophylaxis during the procedure is required for patients with several conditions including prosthetic material used for cardiac valve repair, prior history of infective endocarditis, congenital heart disease, and cardiac transplant patients who develop cardiac vavulopathy (14).

\section{Fasting guideline}

Anesthesiologists must questioned the fasting status of NORA patients; because, patients, parents of pediatric patients, and even proceduralists may not show sufficient attention to this issue. However, fasting status for NORA patients should be similar to classical OR-patients. In this contex, the standard ASA guideline should be followed to avoid the risk of aspiration, except for emergency procedures (Table 3). In pediatrics, clear fluids, such as water and apple/orange juice without particles, can be taken until two hours prior to procedure. This liberal approach avoids hypoglycemia, possible decreased intravascular volume, and irritation of children and their parents (I2).

\section{Choice of anesthetic technique}

Although a small number of NORA procedures is still considered to be performed without assistance of anesthesia experts in some countries of the world, the dominant opinion in this issue is the necessity of an anesthesia care for all procedures. In addition, several clinical indications require a special anesthetic attention, due to the potential risky conditions (Table 4). Many anesthetic techniques including sedation, general anesthesia with endotracheal intubation or laryngeal mask, and regional anesthesia can be used in patients undergoing any interventional procedure outside the OR. Several factors such as type and duration of the procedure, associated medical diseases and patients' health status, and traditional habits of the anesthesia clinic can influense the choice of anesthetic technique. It should be noted that the choice of anaesthetic technique is not directly associated with differences in major outcome parameters (I2).

Standard monitoring of patients undergoing NORA is similar to OR patients, and includes electrocardiography (ECG), blood 
pressure measurement, oxygen saturation, exhaled carbon dioxide and temperature (25). Regular end-tidal carbondioxide $\left(\mathrm{ETCO}_{2}\right)$ measurement is of great importance in the monitorization of patients undergoing NORA, especially those with cardiopulmonary diseases (I5). The ETCO2 monitoring is strongly recommended as an audible alarm for the ventilatory depression associated with the use of propofol to provide deep sedation with or without instrumentation of the airway (4).

Contrary to standard monitorization, procedural medication is different from traditional surgical intervention in terms of many reasons. First of all, most of the procedures are shorter than open surgical interventions. Therefore, anesthetic drugs with rapid onset and offset are generally preffered in NORA procedures $(26,27)$. Tissue trespass is quite less in non-OR procedures compared with classical OR surgeries, which means that most procedures do not result in extensive perioperative pain. Thus, potent, long-acting opioid agents are rarely required in NORA procedures. Neuromuscular blocking agents are only used in patients with endotracheal intubation. Short-acting neuromuscular blockers are, therefore, the preferred choice (I2).

\section{Postprocedural Patient Care}

All patients undergoing a non-OR procedure should be closely monitored during the postprocedural period. Anesthesiologists should be actively involved in the management of airway, oxygenation, ventilation, hemodynamic parameters, and pain control. Possible adverse events such as nausea and vomiting should be also followed up carefully (28). Discharging or transfer of the patient to inpatient clinic or intensive care unit should be decided by the anesthesiologist, in communication with proceduralist.

\section{Morbidity and Mortality in NORA}

Several potential problems related to NORA procedures have been documented (Table 5). Anesthetic complications associated with OR procedures have extensively analyzed to date whereas NORA-related complications have examined in a limited number of clinical studies (29). Hence, current knowledge on NORA-related morbidity and mortality usually comes from closed claims data $(30,31)$. In those studies, NORA patients were older, and had more coexisting systemic diseases, in comparison to OR cases. In addition, emergent cases constitued a higher proportion within overall NORA procedures. In parallel, the proportion of claims for mortality was higher in NORA procedures. When evaluated the cases developed mortality, respiratory events were the leading causes. Specifically, respiratory depression as a result of oversedation was found to be the most common mechanism of injury. Difficult or esophageal intubation and aspiration of gastric contents were the other common complications $(32,33)$. As a result, both loss of airway patency due to airway obstruction and respiratory depression due to a excessive depth of anesthesia/sedation may cause hypoxia and hypercarbia that can result in hypotension and cardiac depression $(28,30,34)$. Administering oxygen during procedure is recommended to reduce the incidence of respiratory complication (35).

Some authors also reported similar mortality rates between OR and NORA procedures in their studies (36). However, more healthier patients, a greater number of short procedures, and a lower proportion of emergency procedures were shown as the possible reasons of this situation by the authors. Interestingly, patients aged under two years had higher mortality rates compared to other patient groups. As known, extremes of age (very young and old) are considered as an independent risk factor for perioperative mortality (37).

In general, periprocedural morbidity or mortality in NORA is associated with three major risk categories including patient-related, procedure-related, and anesthesia-related reasons. Among these, comorbidities or general health status of patients seems to be most frequently observed risk factors $(36,38)$. This data clearly shows the importance of preprocedural evaluation and careful intraprocedural monitoring of the patients undergoing NORA, for minimizing the incidence of unwanted conditions.

\section{CONCLUSION}

Frequency and complexity of NORA procedures are rapidly raising throughout the world. In addition, novel NORA procedures are increasingly introducing routine practice. It is a reality that anesthesia management has a key role for the success of all these procedures. This increases the importance of anesthesiologists in the management of such procedures. Therefore, anesthesia experts, in a closed comminication with other clinicans and proceduralists, should be active in all stages of patient management.

NORA should be considered as a subspeciality of anesthesia, due to its own features and increasing volume. The locations where NORA procedures are performed should be designed to meet all anesthesia needs. A proper and sufficient preprocedural patient evaluation is an important and mandatory step that identifies patient/procedure-related risk factors. Risk stratification algorithms should also include anesthesia/location-related factors. The major endpoint of all these strategies is to improve the patient outcomes, and a multidiciplinary effort by anesthesiologists, proceduralists, other staff personnel, and hospital administrators is necessary to achieve this goal.

Peer-review: Externally peer-reviewed.

Conflict of Interest: Author has no conflicts of interest to declare..

Financial Disclosure: The author declared that this study has received no financial support.

\section{REFERENCES}

I. Kotob F, Twersky RS. Anesthesia outside the operating room: General overview and monitoring standards. Int Anesthesiol Clin 2003; 4I(2): I-15. [Crossref]

2. Youn AM, Ko YK, Kim YH. Anesthesia and sedation outside of the operating room. Korean J Anesthesiol 2015; 68(4): 323-3I. [Crossref]

3. ASA. Statement on nonoperating room anesthetizing locations: committee of origin: standards and practice parameters (approved by the ASA house of delegates on October 19, 1994, and last amended on October 16, 2013). ASA; 2013.

4. ASA. Standards for Basic anesthesia monitoring: committee of origin: standards and practice parameters (approved by the ASA house of delegates on October 21, 1986, last amended on October 20, 2010, and last affirmed on October 28, 2015). ASA; 2015.

5. Evron S, Ezri T. Organizational prerequisites for anesthesia outside the operating room. Curr Opin Anaesthesiology 2009; 22(4): 5I4-8. [Crossref] 
6. Haddy S. Anesthesia for structural heart interventions. Cardiol Clin 20I3; 3I(3): 455-65. [Crossref]

7. Hamid A. Anesthesia for cardiac catheterization procedures. Heart Lung Vessels 20I4; 6(4): 225-31.

8. Dexter F, Wachtel RE. Scheduling for anesthesia at geographic locations remote from the operating room. Curr Opin Anaesthesiol 2014; 27(4): 426-30. [Crossref]

9. Ferrari LR. Anesthesia outside the operating room. Curr Opin Anaesthesiol 2015; 28(4): 439-40. [Crossref]

10. Spahn D. Anaesthesia outside the operating room. Curr Opin Anaesthesiol 2000; 13(4): 407. [Crossref]

II. Aiken LH, Clarkke SP, Cheung RB, Sloane DM, Silber JH. Educational levels of hospital nurses and surgical patient mortality. JAMA 2003; 290(12): 1617-23. [Crossref]

12. Van De Velde M, Kuypers M, Teunkens A, Devroe S. Risk and safety of anesthesia outside the operating room. Minerva Anestesiol 2009; 75(5): 345-8.

13. Bader AM, Correll DJ. Chapter 18: organizational structure of preoperative evaluation center. In: Sweitzer B, editor. Preoperative assessment and management. 2nd edition. Philadelphia: Lippincott, Williams and Wilkins; 2008. p. 420-32.

14. Chang B, Urman RD. Non-operating Room Anesthesia The Principles of Patient Assessment and Preparation. Anesthesiology Clin 2016; 34(I): 223-40. [Crossref]

15. Campbell K, Torres L, Stayer S. Anesthesia and sedation outside the operating room. Anesthesiology Clin 2014; 32(I): 25-43. [Crossref]

16. Weintraub A, Maxwell L. Preoperative assessment and preparation. In: Mattei P, editor. Fundamentals of Pediatric Surgery. New York: Springer; 20II. pp. 3-I5. [Crossref]

17. Spencer C, Franck L. Giving parents written information about children's anesthesia: are setting and timing important? Pediatr Anesth 2005; 15(7): 547-53. [Crossref]

18. Committee on Standards and Practice Parameters, Apfelbaum JL, Connis RT, Nickinovich DG; American Society of Anesthesiologists Task Force on Preanesthesia Evaluation, et al. Practice advisory for preanesthesia evaluation: an updated report by the American Society of Anesthesiologists Task Force on Preanesthesia Evaluation. Anesthesiology 2012; 116: 522-38. [Crossref]

19. Gooden CK, Frost EAM. Preprocedural evaluation: considerations outside of the operating room. Curr Opin Anesthesiol 2015; 28(4): 44I-5. [Crossref]

20. Chetta A, Tzani P, Marangio E, Carbognani P, Bobbio A, Olivieri D. Respiratory effects of surgery and pulmonary function testing in the preoperative evaluation. Acta Biomed 2006; 77(2): 69-74.

21. American Society of Anesthesiologists Committee. Practice Guidelines for Preoperative Fasting and the Use of Pharmacologic Agents to Reduce the Risk of Pulmonary Aspiration: Application to Healthy Patients Undergoing Elective Procedures; An Updated Report by the American Society of Anesthesiologists Committee on Standards and Practice Parameters. Anesthesiology 20II; II4: 4955II. [Crossref]
22. Preibe $H$. Recent advances in preoperative cardiac evaluation. $J$ Curr Pharm Des 2012; 18(38): 6182-94. [Crossref]

23. Fleischer L. Cardiac risk stratification for noncardiac surgery: update from the American College of Cardiology/American Heart Association 2007 guidelines. Cleve Clin J Med 2009; 76(Suppl 4): S9-SI5. [Crossref]

24. Biccard B. Proposed research plan for the derivation of a new cardiac risk index. Anes Analg 2015;120:543-53. [Crossref]

25. Galvagno SM, Kodali BS. Critical monitoring issues outside the operating room. Anesthesiol Clin 2009; 27(I): |4I-56. [Crossref]

26. Tanious MK, Beutler SS, Kaye AD, Urman RD. New hypnotic drug development and pharmacologic considerations for clinical anesthesia. Anesthesiol Clin 2017; 35(2): e95-ell3. [Crossref]

27. Boggs SD, Barnet SR, Urman RD. The future of nonoperating room anesthesia in the 2Ist century: emphasis on quality and safety. Curr Opin Anesthesiol 2017; 30(6): 644-51. [Crossref]

28. Bhavani S. Non-operating room anesthesia in the endoscopy unit. Gastrointest Endosc Clin N Am 2016; 26(3): 47I-83. [Crossref]

29. Melloni C. Morbidity and mortality related to anesthesia outside the operating room. Minerva Anestesiol 2005; 7I(6): 325-34.

30. Robbertze R, Posner KL, Domino KB. Closed claims review of anesthesia for procedures outside the operating room. Curr Opin Anaesthesiol 2006; 19(4): 436-42. [Crossref]

31. Metzner J, Posner KL, Domino KB. The risk and safety of anesthesia at remote locations: the US closed claims analysis. Curr Opin Anaesthesiol 2009; 22(4): 502-8. [Crossref]

32. Bhananker SM, Posner KL, Cheney FW, Caplan RA, Lee LA, Domino KB. Injury and liability associated with monitored anesthesia care: a closed claims analysis. Anesthesiology 2006; 104: 228-34. [Crossref]

33. Metzner J, Domino KB. Risks of anesthesia or sedation outside the operating room: the role of the anesthesia care provider. Curr Opin Anaesthesiol 2010; 23(4): 523-3I. [Crossref]

34. Pambianco DJ. Future directions in endoscopic sedation. Gastrointest Endosc Clin N Am 2008; 18(4): 789-99. [Crossref]

35. Yıldız M, İyilikçi L, Duru S, Hancı $\bigvee$. The attitudes and behaviors of anaesthesiology and reanimation specialists in anaesthesia care applications outside the operating room in Turkey: A Survey Study. Turk J Anaesth Reanim 2014; 42: 196-213. [Crossref]

36. Choi JW, Kim DK, Lee SH, Shin HS, Seong BG. Comparison of safety profiles between non-operating room anesthesia and operating room anesthesia: a study of 199,764 cases at a Korean tertiary hospital. J Korean Med Sci 2018; 33(28): el83. [Crossref]

37. Fecho K, Lunney AT, Boysen PG, Rock P, Norfleet EA. Postoperative mortality after inpatient surgery: incidence and risk factors. Ther Clin Risk Manag 2008; 4(4): 68I-8. [Crossref]

38. Pignaton W, Braz JR, Kusano PS, Módolo MP, de Carvalho LR, Braz MG, et al. Perioperative and anesthesia-related mortality: an 8-year observational survey from a tertiary teaching hospital. Medicine (Baltimore) 2016; 95(2): e2208. [Crossref] 